\title{
Boas práticas executadas pela enfermagem ao paciente submetido a revascularização miocárdica
}

\author{
Good practices performed by nursing to patients undergoing myocardial revascularization \\ Buenas práticas realizadas por enfermeira a pacientes sometidos a revascularización miocárdica
}

Recebido: 19/01/2022 | Revisado: 27/01/2022 | Aceito: 29/01/2022 | Publicado: 31/01/2022

Rodrigo de Albuquerque Lins ORCID: https://orcid.org/0000-0003-2070-792X Universidade Iguaçu, Brasil E-mail: rlins.rl@gmail.com

Kézia Verli Nunes Aredes ORCID: https://orcid.org/0000-0002-4878-8787 Universidade Iguaçu, Brasil

E-mail:kvdanunes@hotmail.com

Bruna Porath Azevedo Fassarella ORCID: https://orcid.org/0000-0002-1400-4147 Universidade Iguaçu, Brasil

E-mail: brunaporath@gmail.com

Larissa Christiny Amorim dos Santos

ORCID: https://orcid.org/0000-0002-9705-5811 Universidade Iguaçu, Brasil

E-mail: amorimlari224@gmail.com

Francine Silva de Souza

ORCID: https://orcid.org/0000-0002-0093-0975

Universidade Iguaçu, Brasil

E-mail: fssbest@hotmail.com

Dayene Rodrigues de Lima

ORCID: https://orcid.org/0000-0002-5287-2949 Universidade Iguaçu, Brasil

E-mail: dazinha_nilo@hotmail.com

Wanderson Alves Ribeiro

ORCID: https://orcid.org/0000-0001-8655-3789 Universidade Iguaçu, Brasil

E-mail: nursing_war@hotmail.com

Keila do Carmo Neves

ORCID: https://orcid.org/0000-0001-6164-1336 Universidade Iguaçu, Brasil

E-mail: keila_arcanjo@hotmail.com

Ana Lúcia Naves Alves

ORCID: https://orcid.org/0000-0003-0791-5775 Universidade Iguaçu, Brasil

E-mail: ananaves.alna@gmail.com

Fernando Salgado do Amaral

ORCID: https://orcid.org/0000-0003-4370-3198 Universidade Iguaçu, Brasil

E-mail: fernando.sal.81@gmail.com

\begin{abstract}
Resumo
Objetivo deste estudo é elucidar através da literatura científica as boas práticas de enfermagem na revascularização do miocárdio. A pesquisa foi de natureza descritiva realizada através de revisão bibliográfica, realizada na base de dados por meio da Biblioteca Virtual em Saúde (BVS), a qual abrange: LILACS, BDENF, Google acadêmico e SCIELO, através dos descritores: Boas práticas, enfermagem e infarto agudo do miocárdio. Os critérios de inclusão foram: obras publicadas na íntegra, em língua portuguesa, publicados nos últimos 10 anos e que possuam aderência ao tema, na qual foram utilizados 21 artigos para construção deste estudo. Compreende-se que, o pós-operatório imediato dos pacientes submetidos à cirurgia cardíaca acontece em Unidade de Terapia Intensiva (UTI), visto que, é um setor que promove recursos suficientes para atender a paciente de alta complexidade, como os que realizam alguma cirurgia cardíaca. Logo, foi possível observar o pós-operatório imediato de pacientes submetidos a revascularização do miocárdio, e a importância da sistematização da assistência de enfermagem como uma tônica na eficácia dos cuidados através do planejamento e preparo seguro, para que toda a equipe a equipe envolvida no processo realize uma abordagem individual e integral ao paciente.
\end{abstract}

Palavras-chave: Boas práticas; Enfermagem; Procedimento cirúrgico cardíaco. 


\begin{abstract}
The aim of this study is to elucidate through the scientific literature the good nursing practices in myocardial revascularization. The research was descriptive in nature, carried out through a literature review, carried out in the database through the Virtual Health Library (VHL), which covers: LILACS, BDENF, academic Google and SCIELO, through the descriptor: good practices and nursing our acute myocardial infarction. The inclusion criteria were: works published in full, in Portuguese, published in the last 10 years and that have adherence to the theme, in which 21 articles were used to build this study. It is understood that the immediate postoperative period of patients undergoing cardiac surgery takes place in the Intensive Care Unit (ICU), since it is a sector that promotes sufficient resources to care for highly complex patients, such as those who undergo surgery cardiac arrest. Therefore, it was possible to observe the immediate postoperative period of patients undergoing myocardial revascularization, and the importance of systematizing nursing care as a tonic in the effectiveness of care through planning and safe preparation, so that the entire team involved in the process takes an individual and integral approach to the patient.
\end{abstract}

Keywords: Good habits; Nursing; Cardiac surgical procedure.

\title{
Resumen
}

El objetivo de este estudio es dilucidar a través de la literatura científica las buenas prácticas de enfermería en la revascularización miocárdica. La investigación fue de carácter descriptivo, realizada a través de una revisión bibliográfica, realizada en la base de datos a través de la Biblioteca Virtual en Salud (BVS), que abarca: LILACS, BDENF, Google académico y SCIELO, a través de los descriptores: buenas prácticas y nuestros agudos de enfermería. infarto de miocardio. Los criterios de inclusión fueron: trabajos publicados íntegramente, en portugués, publicados en los últimos 10 años y que adhieran a la temática, en los que se utilizaron 21 artículos para la construcción de este estudio. Se entiende que el postoperatorio inmediato de los pacientes sometidos a cirugía cardiaca se desarrolla en la Unidad de Cuidados Intensivos (UCI), ya que es un sector que promueve recursos suficientes para atender a pacientes de alta complejidad, como los que se someten a cirugía cardiaca. Por lo tanto, fue posible observar el postoperatorio inmediato de los pacientes sometidos a revascularización miocárdica, y la importancia de la sistematización de los cuidados de enfermería como clave para la eficacia de los cuidados a través de la planificación y preparación segura, para que todo el equipo involucrado en el proceso realice un enfoque individual e integral del paciente.

Palabras clave: Buenas practicas; Enfermería; Procedimiento quirúrgico cardíaco.

\section{Introdução}

Segundo as estimativas da Organização Mundial da Saúde, 17,9 milhões de pessoas morreram de doenças cardiovasculares em 2016, representando 31\% de todas as mortes globais. Parte destas enfermidades determinam tratamentos cirúrgicos, como as cirurgias de revascularização do miocárdio, correção de doenças valvares, correção de doenças da aorta e de cardiopatias congénitas, as mais comuns em adultos e idosos, com vista ao estilo de vida que a sociedade tem vivenciado. Estes pacientes, costumam serem encaminhados para centro cirúrgico com maior número de comorbidades, passiveis complicações no pós-operatório, o que ocasiona um aumento do tempo de internamento hospitalar e tecnológicos investidos no seu tratamento e recuperação (Lopes et al., 2019).

Corrobora-se que 17,5 milhões de pessoas faleceram de doenças cardiovasculares em 2012, representando $31 \%$ de todas as mortes mundial. Desses óbitos, cerca de 7,4 milhões foram devidos à doença arterial coronariana (DAC). No Brasil, as doenças cardiovasculares seguem padrão semelhante, configurando-se como a principal causa de óbito, e a prevalência de DAC na população adulta está estimada em 5 a $8 \%$ (Silveira et al., 2018).

A idade influencia para a evolução das taxas de incidência e mortalidade das doenças coronarianas, além de estarem relacionadas às modificações quanto ao estilo de vida, que influenciam simultaneamente todas as faixas etárias (Santos et al., 2018).

Embora os avanços tecnológicos na cirurgia cardíaca e nos cuidados pré-operatórios, as complicações pós-operatórias são frequentes, levando a um aumento substancial na mortalidade de pacientes submetidos a esses procedimentos cirúrgicos (Reis et al., 2019). Sendo um procedimento invasivo de alto risco e amplamente difundido mundialmente como terapêutica de escolha para diversas patologias do aparelho cardiovascular, em distintas faixas etárias (Lima et al., 2019). 
O período pré-operatório é proposto ao processo de preparo do paciente como um todo, bem como, é um período pertinente ao processo educativo de oferecer informações a respeito da maneira como são realizados os procedimentos cirúrgicos e todo dos cuidados a serem realizados (Gomes; Silva; Bezerra, 2020).

Conforme Rabelo et al., (2017, p. 2)

A cirurgia cardíaca é indicada quando a probabilidade de sobrevida é maior com o tratamento cirúrgico do que com o tratamento clínico. É considerada de grande porte e, para a sua realização, na maioria das vezes, faz-se necessária a utilização da circulação extracorpórea. Assim, pacientes submetidos à cirurgia cardíaca necessitam de cuidados intensivos e são encaminhados para uma Unidade de Terapia Intensiva (UTI) no Pós-Operatório Imediato (POI).

O período POI é o período de recuperação crítica que recomenda a necessidade de cuidados intensivos, com a desígnio de se estabelecer uma boa recuperação dos pacientes. As repercussões orgânicas do procedimento e os mecanismos fisiológicos precisam ser verificados, como possíveis complicações destes processos. Sob a ótica da enfermagem, dependerá um modelo conceptual disciplinar, as pessoas são consideradas como sistemas abertos mantendo interação contínua com o ambiente, estando expostas a diversos estímulos que provocam um processo de coping capaz de resultar em respostas adaptativas ou ineficazes face à estratégia gerada pelo estímulo da cirurgia cardíaca (Lopes et al., 2019).

Segundo Kahl et al. (2019, p. 6) os cuidados de enfermagem ao paciente no pós operatório de cirurgia cardíaca abrangem desde os registros completos em prontuários, avaliação da incisão cirurgia (presença de exsudato e sinais flogísticos, avaliação hemodinâmica, dentre outros.

O cuidado pós-operatório deve ser realizado de forma tão criteriosa quanto o pré-operatório e o transoperatório. A equipe de enfermagem necessita atentar as manifestações clínicas do indivíduo, conhecer sua história pregressa e a evolução do tratamento em todos os períodos. São comuns complicações decorrentes da instabilidade hemodinâmica ou sintomas de incapacidade cardiorrespiratória, como o infarto agudo do miocárdio (IAM), insuficiência cardíaca congestiva (ICC), hipertensão arterial sistêmica (HAS) e hipertensão pulmonar, arritmias, dificuldades respiratórias, cerebrovasculares, neurológicas, infecciosas e as renais (Silva et al., 2017) (Reis et al., 2019).

A Sistematização da Assistência de Enfermagem (SAE) é de extrema importância no pós-operatório, tendo em vistas as modificações fisiológicas potenciais e reais, o que sanciona que a equipe de Enfermagem necessita estar atenta às alterações hemodinâmicas e condições que promovam estabilidade do paciente. Logo, os cuidados são estabelecidos conforme as necessidades dos pacientes, visando à manutenção do equilíbrio hemodinâmico e das suas funções vitais (Ribeiro et al., 2015).

Destaca-se que a atuação do enfermeiro na fase pós-operatória que costuma causar certa preocupação nas primeiras 24h, é realizada desde a avaliação a recuperação dos efeitos anestésicos, avaliação frequente do estado fisiológico, monitoração de complicações, ventilação apropriada, estabilidade hemodinâmica, integridade do sítio cirúrgico, náuseas e vômitos e estado neurológico (Smeltzer; Bare, 2014).

As principais complicações identificadas no pós operatório são voltadas para o fator de risco não modificável histórico familiar de cardiopatias, risco modificável dislipidemia, circulação extra corpórea (CEC) por levar o organismo a uma resposta inflamatória sistêmica, causada pela circulação do sangue por meio do circuito de extracorpórea e pela formação de microêmbolos (Andrade et al., 2019).

A escolha desta temática vem ao encontro a busca pelo aperfeiçoamento a qualidade da assistência de enfermagem prestada, o enfermeiro deve organizar e planejar o cuidado a partir da aplicação das etapas metodológicas do processo de enfermagem, de maneira a intervir de acordo com as necessidades do paciente, afim de promover sua recuperação mais rápido possível e desospitalização precoce (Taurino, 2019). 
Conforme Cabral; Chaves (2020, p. 2) o processo de trabalho de enfermagem na UTI, sobretudo com o paciente cardiopata no pós-operatório é marcado por atividades assistenciais complexas que exigem alta capacidade técnica e científica, afinal, a tomada de decisões imediatas e a adoção de condutas seguras estão diretamente relacionadas à vida e a morte dos pacientes. Logo, é necessário que os acadêmicos e os profissionais atuantes sejam qualificados adequadamente dimensionado para desenvolver a assistência com qualidade e segurança.

A prática assistencial deve ser pautada no método científico, considerando as prioridades distintas de acordo com o período do pós-operatório, ou seja, se imediato, mediato ou tardio. Para atendê-las adequadamente, o enfermeiro necessita desenvolver habilidades e competência cognitivas, técnicas, organizacionais e de relação interpessoal construtiva, ponderando que ora poderão ter caráter objetivo e ora subjetivo (Taurino, 2019).

As boas práticas de enfermagem realizadas a pacientes submetidos a revascularização do miocárdio têm sido suficientes para seu processo de recuperação? Para responder à questão norteadora foram criados os objetivos elucidar através da literatura científica as boas práticas de enfermagem na revascularização do miocárdio, apontar boas práticas implementadas ao paciente submetido a revascularização cardíaca e descrever as ações executadas pela equipe de enfermagem para a recuperação pós operatória.

\section{Metodologia}

A pesquisa é caracterizada por ser um estudo descritivo, realizada através de revisão bibliográfica. Sendo esta, essencial para todas as pesquisas, pois, admite explicitar os objetivos em termos do que já foi pesquisado e escrito, ou debater os resultados das pesquisas anteriores e seus impactos no campo científico. É composto por: formulação de uma questão que embase a revisão; modos de identificação de estudos relevantes que possam agregar a revisão; seleção de estudos relevantes; a análise crítica da qualidade da metodologia de pesquisa dos estudos selecionados para compor a revisão; e a síntese dos resultados nos estudos escolhidos para a revisão (Galvão et al., 2018).

A revisão da literatura auxilia na delimitação do problema da pesquisa e busca de novas linhas de investigação para o problema a ser investigado, evita que o pesquisador procure caminhos nunca percorridos, identificar trabalhos já concretizados e escritos a partir de outra abordagem e evita que o pesquisador faça mais do mesmo, que diga o que já foi relatado, tornando a sua pesquisa irrelevante (Brizola \& Fantin, 2016).

Para a construção desta pesquisa, foi coletado dados entre março a setembro de 2021. A pesquisa foi realizada na base de dados por meio da Biblioteca Virtual em Saúde (BVS), a qual abrange: LILACS, BDENF, Google acadêmico e SCIELO, através do descritor: Boas práticas, enfermagem e infarto agudo do miocárdio.

Os critérios de inclusão foram: obras publicadas na íntegra, em língua portuguesa, publicados nos últimos 10 anos e que possuam aderência ao tema e como critérios de exclusão foram: obras que ultrapassem 10 anos, em língua estrangeira e estudos que não abordassem a temática relevante ao objetivo desse assunto. 


\section{Resultados e Discussão}

Tabela 1. Análise da busca:

\begin{tabular}{c|c|c}
\hline Palavras-Chave & Sites pesquisados & Artigos pós seleção de critérios \\
\hline boas práticas and enfermagem & BDENF -140 artigos & 06 \\
\cline { 2 - 3 } our infarto agudo do miocárdio & LILACS - 165 artigos & 09 \\
\cline { 2 - 3 } & SCIELO - 90 artigos & 06 \\
\hline & Google Acadêmico- 4.400 & 01 \\
\hline
\end{tabular}

Fonte: Autores.

De acordo com o resultado da pesquisa bibliográfica, realizada para que se atenda o objetivo da pesquisa, obteve-se o total de 21 artigos lidos na íntegra, as quais estão representados na tabela abaixo:

Quadro 1 - Demonstrativo dos artigos encontrados relacionando os autores e ano, título, local de publicação dos periódicos, base de dados.

\begin{tabular}{|c|c|c|c|}
\hline Autores/ ano & Título do artigo & Base de dados & Tipos de metodologia \\
\hline $\begin{array}{l}\text { Almeida, P. S, et al., } \\
2017 .\end{array}$ & $\begin{array}{l}\text { Implementação de orientações de enfermagem } \\
\text { aos pacientes pré-operatórios de cirurgia } \\
\text { cardíaca em meio digital. }\end{array}$ & SCIELO & Ensaio clínico randomizado. \\
\hline $\begin{array}{l}\text { Amorim, T. V; } \\
\text { Salimena, A. M. O, } \\
2015 .\end{array}$ & $\begin{array}{l}\text { Processo cirúrgico cardíaco e suas implicações } \\
\text { no cuidado de enfermagem: revisão/reflexão. }\end{array}$ & LILACS & Revisão integrativa. \\
\hline $\begin{array}{l}\text { Andrade, A. Y. T, et } \\
\text { al., } 2019 .\end{array}$ & $\begin{array}{l}\text { Complicações no pós-operatório imediato de } \\
\text { revascularização do miocárdio. }\end{array}$ & BDENF & Revisão integrativa. \\
\hline $\begin{array}{l}\text { Barretta, J. C, et al., } \\
2017 .\end{array}$ & $\begin{array}{l}\text { Pós-operatório em cirurgia cardíaca: refletindo } \\
\text { sobre o cuidado de enfermagem. }\end{array}$ & BDENF & Revisão integrativa. \\
\hline $\begin{array}{l}\text { Branco, C. S. P. C; } \\
\text { Pereira, H, } 2016 .\end{array}$ & $\begin{array}{llll}\text { Cuidados de enfermagem ao paciente em } & \text { pós } \\
\text { operatório imediato de cirurgia } & \text { de } \\
\text { revascularização do miocárdio. } & & \end{array}$ & LILACS & Revisão de literatura. \\
\hline $\begin{array}{l}\text { Camponogara, } \mathrm{S}, \text { et } \\
\text { al., } 2012 .\end{array}$ & $\begin{array}{l}\text { Percepção de pacientes sobre o período pré- } \\
\text { operatório de cirurgia cardíaca. }\end{array}$ & SCIELO & $\begin{array}{l}\text { Entrevista semiestruturada e análise de } \\
\text { conteúdo. }\end{array}$ \\
\hline $\begin{array}{l}\text { Coppetti, L. C; } \\
\text { Stumm, E. M. F; } \\
\text { Benetti, E. R. R, } \\
2015 .\end{array}$ & $\begin{array}{l}\text { Considerações de pacientes no perioperatório de } \\
\text { cirurgia cardíaca referentes às orientações } \\
\text { recebidas do enfermeiro. }\end{array}$ & LILACS & Investigação de abordagem qualitativa. \\
\hline $\begin{array}{l}\text { Garcia, R. P, et al., } \\
2015 .\end{array}$ & $\begin{array}{l}\text { Apoio social frente à necessidade de cuidado } \\
\text { após infarto do miocárdio. }\end{array}$ & LILACS & Qualitativa e exploratória. \\
\hline $\begin{array}{l}\text { Lima, V. R; Leal, C. } \\
\text { C. G; Flávio, A. G. C, } \\
2014 .\end{array}$ & $\begin{array}{l}\text { Complicações pós-operatórias em idosos } \\
\text { submetidos à revascularização do miocárdio. }\end{array}$ & SCIELO & Revisão bibliográfica. \\
\hline $\begin{array}{l}\text { Matioli, K. B. B, et } \\
\text { al., 2021. }\end{array}$ & $\begin{array}{l}\text { Delirium: prevalência e fatores associados ao } \\
\text { pós-operatório de cirurgia cardiovascular em } \\
\text { idosos. }\end{array}$ & BDENF & $\begin{array}{l}\text { Estudo quantitativa e caráter transversal } \\
\text { e analítico. }\end{array}$ \\
\hline $\begin{array}{l}\text { Menezes, T. C, et al., } \\
2018\end{array}$ & $\begin{array}{l}\text { Comparações e correlações da intensidade da dor } \\
\text { e da força muscular periférica e respiratória no } \\
\text { pré e pós-operatório de cirurgia cardíaca. }\end{array}$ & LILACS & Estudo observacional longitudinal. \\
\hline
\end{tabular}




\begin{tabular}{|c|c|c|c|}
\hline $\begin{array}{l}\text { Paiva, A. C. R, et al., } \\
2015 .\end{array}$ & $\begin{array}{l}\text { Checklist de cirurgia segura: análise do } \\
\text { preenchimento da ficha de verificação no pré, } \\
\text { trans e pós-operatório. }\end{array}$ & $\begin{array}{c}\text { Google } \\
\text { Acadêmico }\end{array}$ & $\begin{array}{l}\text { Relato de experiência elaborado pelos } \\
\text { discentes do } 7 \text { período de graduação em } \\
\text { Enfermagem de uma Universidade. }\end{array}$ \\
\hline $\begin{array}{l}\text { Passinho, R. S, et al., } \\
2019 .\end{array}$ & $\begin{array}{l}\text { Sinais, sintomas e complicações do infarto agudo } \\
\text { do miocárdio. }\end{array}$ & LILACS & Revisão integrativa. \\
\hline $\begin{array}{lllll}\text { Paz, } & \text { V. } & \text { P. et } & \text { al., } \\
2019 . & & & & \end{array}$ & $\begin{array}{l}\text { Sistemas de cuidados à saúde de pessoas com } \\
\text { infarto do miocárdio: revisão da literatura. }\end{array}$ & BDENF & Revisão integrativa. \\
\hline $\begin{array}{l}\text { Pereira, K. S. M, et } \\
\text { al., 2016. }\end{array}$ & Complicações cardíacas em cirurgia vascular. & SCIELO & $\begin{array}{l}\text { Estudo prospecto de } 200 \text { pacientes } \\
\text { submetidos a cirurgia vascular arterial. }\end{array}$ \\
\hline $\begin{array}{l}\text { Ribeiro, C. P et al., } \\
2018 .\end{array}$ & $\begin{array}{l}\text { Diagnósticos de enfermagem em pacientes no } \\
\text { pós-operatório de cirurgia cardíaca. }\end{array}$ & SCIELO & $\begin{array}{l}\text { Estudo descritivo, com delineamento } \\
\text { transversal e abordagem quantitativa. }\end{array}$ \\
\hline $\begin{array}{l}\text { Rosa, R. S, } \\
\text { et al., } 2016 .\end{array}$ & $\begin{array}{l}\text { Evidências para o cuidado de enfermagem na } \\
\text { avaliação do risco coronariano em pacientes } \\
\text { hospitalizados. }\end{array}$ & SCIELO & Estudo descritivo de corte transversa. \\
\hline $\begin{array}{l}\text { Silva, C. L. D, } \\
\text { et al., } 2018 .\end{array}$ & $\begin{array}{l}\text { Intervenções de enfermagem em pacientes da } \\
\text { unidade de terapia intensiva cardiológica de um } \\
\text { hospital universitário submetidos à cirurgia de } \\
\text { revascularização do miocárdio. }\end{array}$ & LILACS & $\begin{array}{l}\text { Estudo descritivo, retrospectivo, } \\
\text { transversal, documental, } \\
\text { quantitativa. }\end{array}$ \\
\hline $\begin{array}{l}\text { Silva, C. J. B, et al., } \\
2016 .\end{array}$ & $\begin{array}{l}\text { Banho no leito de infartados: crossover do } \\
\text { controle hidrotérmico } 40^{\circ} \mathrm{C} \text { versus } 42,5^{\circ} \mathrm{C} \text {. }\end{array}$ & LILACS & Estudo de caso, qualitativo. \\
\hline $\begin{array}{l}\text { Silva L. L. T, et al., } \\
2017 .\end{array}$ & $\begin{array}{l}\text { Cuidados de enfermagem nas complicações no } \\
\text { pós-operatório de cirurgia de revascularização } \\
\text { do miocárdio. }\end{array}$ & LILACS & Estudo descritivo retrospectivo. \\
\hline $\begin{array}{l}\text { Silva, M. T; Palu, L. } \\
\text { A; Brusamarello, T, } \\
2018 .\end{array}$ & $\begin{array}{l}\text { Prevenção de complicações evitáveis em uma } \\
\text { unidade de terapia intensiva: uma revisão } \\
\text { integrativa. }\end{array}$ & BDENF & $\begin{array}{l}\text { Revisão } \\
\text { Integrativa. }\end{array}$ \\
\hline
\end{tabular}

Fonte: Autores.

\subsection{Infarto agudo do miocárdio}

Pereira et al. (2016, p. 8) afirma que as diretrizes sugerem que o risco médio de IAM no perioperatório ou morte cardíaca está relacionado com o reconhecimento prioritário dos fatores clínicos, tais como: insuficiência cardíaca, angina, IAM, idade avançada, capacidade funcional e, para os pacientes submetidos a exames não invasivos, que tenham os territórios isquêmicos do miocárdio demonstrados através de testes provocativos. O grau de risco conferido por um procedimento cirúrgico não cardíaco em acréscimo aos fatores de risco paciente-específico tem apresentado dificuldade na sua elucidação. Por maior que seja as pesquisas em cirurgia vascular até o momento, acredita-se que os riscos cirúrgicos envolvem a associação entre a DAP e débito cardíaco (DC).

No presente estudo avaliou que existem pacientes hospitalizados em pós operatório de cirurgia cardíaca em risco coronariano, possuem risco de desenvolver um IAM de acordo os critérios do escore de framingham, ficando evidente que o risco maior ocorre devido a idade avançada, bem como as comorbidades: hipertensão, diabetes e DAC (Rosa et al., 2016).

Passinho et al. (2018, p. 9) pontua que é necessário que o enfermeiro compreenda as manifestações clínicas peculiares em seu estudo destacou as taquicardias e bradicardias análogos anginosos da isquemia miocárdica. A bradicardia ocorre devido à redução do ritmo cardíaco durante a isquemia (resultado da hiperatividade do sistema nervoso parassimpático); baixo débito cardíaco como síncope ou pré-síncope; tontura; sensação de cabeça vazia; fraqueza e fadiga (diminuição na capacidade de bombeamento do coração); congestão pulmonar; insuficiência cardíaca; dentre outros. Em casos de taquicardia é acompanha a dor torácica e hiperatividade do sistema nervoso simpático). A dor esofágica ocorre devido o refluxo de ácido no estômago, espasmo e obstrução, bem como, a dor abdominal estar pertinente a parede inferior durante o infarto de ventrículo direito. 
Logo, a adoção dos cuidados necessita ser elaborada e implementada no cotidiano do enfermeiro quando o objetivo de reduzir a morbidade e mortalidade acarretada pelas doenças cardiovasculares. Parte dos pacientes hospitalizados não têm ciência sobre a presença de fatores risco cardiovascular no seu dia-a-dia, muito menos que podem causar consequências manifestos na sua qualidade de vida, e que posteriormente possam vir desenvolver um evento coronariano. É notório que muitos desses fatores passam despercebidos por parte dos enfermeiros que atuam nas instituições de saúde (Rosa et al., 2016).

\subsection{Ações de enfermagem para recuperação pós operatória}

Amorim; Salimena (2015, p. 4) destaca três tipos de cirurgia cardíaca: corretoras (fechamento de canal arterial, como exemplo, defeito de septo atrial e ventricular), reconstrutoras (revascularização do miocárdio, plastia de valva aórtica, mitral ou tricúspide) e substitutivas (trocas valvares e transplantes, a exemplo, Revascularização do Miocárdio).

A cirurgia cardíaca surgiu concomitante a circulação extracorpórea, é um marco para área da saúde, visto que a partir dele foi possível a manipulação direta do coração, facilitando a correção de inúmeras patologias cardíacas antes consideradas incuráveis. É realizado através de um sistema de máquinas, aparelhos e circuitos ligados ao coração do paciente, com a finalidade de suprir as funções cardíacas e pulmonares, poupando a integridade celular, a estrutura, função e metabolismo dos demais órgãos do paciente (Barreta et al., 2017).

A Cirurgia de Revascularização do Miocárdio (CRVM) é uma cirurgia tipo reconstrutora que consiste em um implante de um enxerto arterial coronário, empregando a veia safena ou a artéria mamária interna e em alguns casos a artéria radial. Seu desígnio é isolar a artéria ocluída e restaurar a perfusão da artéria coronária lesionada, preservando dessa maneira o miocárdio (Branco; Pereira, 2016).

O tipo de cirurgia mais empregada é a CRM na qual é realizada técnica-padrão, o coração seja parado e a circulação seja conservada através da Circulação Extracorpórea (CEC). O que exige da equipe de saúde ações que buscam garantir ao paciente uma assistência de qualidade resultando numa rápida recuperação e desospitalização precoce (Barreta et al., 2017).

A cirurgia de CRM é recomendada quando a probabilidade de uma vida útil é maior que o tratamento clínico. É um dos procedimentos cirúrgicos que mais mobilizam o paciente, visto que, é considerado altamente complexa, levando os pacientes cardiopatas a atribuírem sentimentos de medo e sensação de morte (Camponogara et al., 2012).

Almeida et al. (2017, p. 2) pontua em seu estudo que a CRM é uma intervenção que ajuda no alívio dos sintomas, consentindo o restabelecimento da condição física, aumentando a sobrevida e promovendo maior qualidade de vida ao indivíduo.

Com vistas as intervenções de enfermagem, são fundamentais para a recuperação do paciente submetido à cirurgia e, baseado seus conhecimentos e implementado novas escolhas de assistência, por meio de uma metodologia adequada de trabalho, definida pela SAE. Está sendo implementada na prática assistencial e, afere maior segurança aos pacientes, melhora a qualidade da assistência e a autonomia aos profissionais, por estabelecer o trabalho quanto ao método, pessoal e instrumentos e viabilizar a operacionalização do Processo de Enfermagem (Ribeiro et al., 2015).

Coppetti; Stumm; Benetti (2015, p. 5) acrescenta que os pacientes costumam sentir ansiedade e angustia, esses sentimentos estão relacionados à carência de conhecimento referente à doença, ao procedimento cirúrgico e à recuperação. Ainda, o estresse do paciente no pré-operatório relaciona-se à desinformação acerca dos procedimentos cirúrgicos e os relativos cuidados no pós-operatório.

No Brasil no ano de 2011, foram realizadas aproximadamente 100 mil cirurgias cardíacas, dentre essas, 50 mil com circulação extracorpórea (CEC) e mais da metade para revascularização miocárdica em mais de 170 centros distribuídos em todos os Estados Brasileiros com a participação de mais de 1000 cirurgiões associados à Sociedade Brasileira de Cirurgia Cardiovascular (Barreta et al., 2017). 
Paiva et al., (2015, p. 3) destaca que para toda e qualquer cirurgia, independentemente do seu grau de complexidade, é realizada uma lista de verificação de segurança cirúrgica (checklist). O uso dessa ferramenta visa melhorar a assistência cirúrgica, por meio de padrões de segurança no pré, trans e pós-operatório, sendo o enfermeiro o profissional indicado para orientar a checagem dos dados do paciente, informações clínicas e funcionamento dos equipamentos, podendo prevenir uma série de complicações durante o procedimento.

O tempo cirúrgico costuma variar entre 4 e 16 horas, com média de 9 horas e 20 minutos, as complicações pósoperatórias descritas são: arritmias, hipotensão, fibrilação atrial, síndrome do baixo débito cardíaco, parada cardiorrespiratória, sangramento, instabilidade hemodinâmica, hipoxemia e pneumotórax (Andrade et al., 2019).

A abertura do tórax durante a cirurgia cardíaca pode atingir a inervação e a musculatura respiratória, comprometendo a Força da Musculatura Respiratória (FMR), logo, acometendo o padrão respiratório desses pacientes, faz-se necessário reduzir a ansiedade e ofertar conforto no leito no período pré-operatório, para que não acarrete prolongamento da ventilação mecânica no período PO (Menezes et al., 2018).

Em pacientes de cirurgia cardíaca a redução da FMR tem sido associada com diminuição da capacidade funcional e colaborando para um momento prolongado de recuperação da função pulmonar e episódio de perda do condicionamento físico, que pode estender-se por muitas semanas. As repercussões respiratórias também provocam modificações na FMR, assim como modificações nos volumes e capacidades pulmonares, disfunção alveolar, depressão do estímulo respiratório central e distúrbios mecânicos da função torácica (Menezes et al., 2018).

\subsection{Boas práticas de enfermagem no pós operatório de revascularização do miocárdio em UTI}

Paz et al., (2019, P. 8) compara em seu estudo a busca de atendimento entre homens e mulheres no sistema formal de saúde, e percebeu-se que as mulheres adiaram mais tempo para realizarem a cirurgia. Esse achado está pertinente ao fato de as mulheres não quererem preocupar a família, ao medo de se ausentarem para o procedimento cirúrgico. Esse contexto cultural pode comprometer significativamente o agravamento da doença, aumento de ansiedade para a alta e comprometer o planejamento de cuidados do enfermeiro (Garcia et al., 2015).

Lima; Leal; Flávio (2014, p.7) também salienta que o comportamento dos pacientes no pós-operatório compromete diretamente no cuidar. Logo, cabe ao enfermeiro realizar o planejamento individualizado, com raciocínio clínico diretamente na prevenção e promoção dos cuidados ao paciente operado, evidenciando qualquer complicação pós-operatória, impedindo maiores agravos ao paciente no período de convalescença.

Compreende-se que, o pós-operatório imediato dos pacientes submetidos à cirurgia cardíaca acontece em UTI, visto que, é um setor que promove recursos suficientes para atender a paciente de alta complexidade, como os que realizam alguma cirurgia cardíaca.

Atuação do enfermeiro a paciente submetido à cirurgia cardíaca no pós-operatório imediato se inicia no centro cirúrgico, é realizado ações ligeiras e sincronizadas desde a instalação da ventilação mecânica, monitorização cardíaca, aquecimento do cliente, acoplamento dos drenos torácicos aos frascos de drenagem, controle da diurese horária, administração de líquidos infundidos, avaliação permanente do nível de consciência e de dor (Barreta et al., 2017).

O período do pós-operatório imediato (POI) compreende as primeiras 24 horas após o término da cirurgia. Os cuidados de pós-operatório imediato são: manutenção do débito cardíaco, a diminuição de dor, perfusão tissular adequada, integridade tecidual, equilíbrio hidroeletrolítico e controle da glicemia, manutenção da ventilação e oxigenação adequadas, precauções e medidas para redução de risco de infecção, comunicação, redução da ansiedade do paciente e de seus familiares (Pereira et al., 2016) (Silva et al., 2017). 
Matioli et al., (2021, p. 5) demonstra em seu estudo que é comum o delirium em pacientes no pós-operatório cardíaco, bem como, risco elevado de desenvolver arritmias, doenças renais e insuficiência cardíaca congestiva, acarretando mortalidade. Neste estudo dos 50 participantes que compuseram a população de amostra, $40 \%$ apresentaram ao menos um episódio durante a internação em ambiente de cuidados intensivos.

O enfermeiro deve direcionar seus cuidados a ventilação mecânica, monitoração cardíaca, controle de diurese e pressão arterial, administração de líquidos infundidos, avaliação do nível de consciência e de dor, manutenção da integridade tecidual, prevenção e controle de infecção, lavagem de mãos, administração de medicamentos prescritos e escuta do paciente (Barreta et al., 2017).

Silva et al. (2017, p. 1) acrescenta os cuidados as possíveis complicações renais existentes, é necessário controle do balanço hídrico e o monitoramento minucioso da função renal. Os cuidados neurológicos incluem verificação da responsividade, orientação ao paciente no tempo e no espaço e utilização da Escala de Coma de Glasgow. Quanto ao respiratório são: melhoria da amplitude respiratória, monitoração da oximetria de pulso, manutenção da frequência respiratória normal, avaliação da causa da alteração e medidas para melhorar o padrão respiratório (Silva et al., 2017).

As boas práticas de enfermagem em UTI são voltadas para às necessidades fisiológicas e psicossociais como o conforto, sono e repouso, alívio da dor, manutenção do equilíbrio eletrolítico, regulação da temperatura, eliminação vesico intestinal, movimentos, exercícios, alimentação e drenos (Amorim; Salimena, 2015).

Silva et al., (2016, p. 4) afirma que, o banho durante o período de repouso, tem a finalidade de promover higiene, regulação térmica, diminuição da microbiota, satisfação, conforto e comodidade. Para a efetivação efetiva e segura desse cuidado, é imprescindível que os profissionais sejam capacitados para identificar intercorrências, já que as mudanças do posicionamento na cama, alteração térmica da água e ambiente e o descondicionamento clínico podem impactar na resposta hemodinâmica do paciente.

Em UTI as condições clínicas do paciente oscilam entre limites estreitos de normalidade/anormalidade, na qual pequenas mudanças orgânicas podem levar à deterioração grave da função corporal. A boa pratica de enfermagem para não ocorrência de erros é sem dúvida, direcionada a segurança na assistência (Silva et al., 2018).

Silva; Palu; Brusamarello (2018, p. 2) destacam: prevenção de erros nas dosagens de medicações (uso de checklist e/ou protocolos para evitar falhas nas programações e acompanhamentos das infusões); prevenção referente ao cuidado das vias aéreas (cuidados bucais com aspiração e higiene oral, cabeça da cama elevada); prevenção de lesão por pressão (reposicionamento do paciente no leito, uso de colchões especiais, avaliação da pele por meio da escala de Braden); dentre outros.

\section{Considerações Finais}

Com este estudo, foi possível observar o pós-operatório imediato de pacientes submetidos a revascularização do miocárdio, e a importância da sistematização da assistência de enfermagem como uma tônica na eficácia dos cuidados através do planejamento e preparo seguro, para que toda a equipe a equipe envolvida no processo realize uma abordagem individual e integral ao paciente.

Mediante a isto, a enfermagem tem grande contribuição e influência direta na rotina pós operatória quanto a desospitalização o mais breve possível da unidade hospitalar, com vistas, ao crescente número de procedimentos cirúrgicos realizados anualmente e aos elevados índices de complicações associadas a eles. Este estudo permitiu uma melhor compreensão das práticas assistenciais ofertadas pelos profissionais de enfermagem que atuam na assistência ao paciente no pós cirúrgico cardíaco. 
Acredita-se que, a realização de treinamentos peculiares pode melhorar o modo de execução das práticas propostas pelo enfermeiro, para que tais condutas caminhem para um processo terapêutico de qualidade, a fim de atentá-los para a manutenção de conhecimentos acerca dos cuidados e tratamento clínico, com base nas necessidades do paciente e complexidade do procedimento.

Torna-se sugestível a ampliação deste estudo, visando maior divulgação para a população sobre esta prática, não só pela relevância científica que a temática propõe, mas também a relevância social para que possa melhorar as práticas de enfermagem oferecida aos pacientes submetidos a uma cirurgia de complexidade e que pode acarretar morbidade quando ocorre falha(s) em algum processo assistencial.

Faz-se necessário que tenham novos estudos sobre este tema, pois ainda a lacunas existentes, tais como a falta de relação com as condutas apropriadas, preconizadas e fundamentadas em evidências científicas, a busca pelo potencial e limitações dos profissionais, a fim de diminuir as comorbidades pertinentes aos cuidados realizados no pós operatório do cirúrgico.

\section{Referências}

Almeida, P. S., Pellanda, L. C., Caregnato, R. C. A., \& Souza, E. N. (2017). Implementação de orientações de enfermagem aos pacientes pré-operatórios de cirurgia cardíaca em meio digital. Rev. SOBECC, 68-75.

Amorim, T. V., \& Oliveira, S. A. M. (2015). Processo cirúrgico cardíaco e suas implicações no cuidado de enfermagem: reflexão. HU Revista, 41(3 e 4).

Andrade, A. Y. T. D., Tanaka, P. S. D. L., Poveda, V. D. B., \& Turrini, R. N. T. (2019). Complicações no pós-operatório imediato de revascularização do miocárdio. Rev. SOBECC, 224-230.

Barretta, J. C., Auda, J. M., Antoniolli, D., \& Barancelli, M. D. C. (2017). Pós-operatório em cirurgia cardíaca: refletindo sobre o cuidado de enfermagem Postoperative in cardiac surgery: reflecting about nursing care. Revista de Pesquisa Cuidado é Fundamental Online, 9(1), 259-264.

Branco, C. D. S. P. C., \& Pereira, H. O. (2016). Cuidados de enfermagem ao paciente em pós operatório imediato de cirurgia de revascularização do miocárdio. Enfermagem Revista, 19(1), 72-84.

Brizola, J., \& Fantin, N. (2016). Revisão da literatura e revisão sistemática da literatura. Revista de Educação do Vale do Arinos-RELVA, 3(2).

Cabral, J. V. B., \& de Castro, C. J. S. (2020). Cuidado de enfermagem no pós-operatório de cirurgia cardíaca pediátrica: revisão integrativa. Revista Enfermagem Contemporânea, 9(1), 118-126.

Camponogara, S., Soares, S. G. A., Silveira, M., Viero, C. M., Barros, C. S. D., \& Cielo, C. (2012). Percepção de pacientes sobre o período pré-operatório de cirurgia cardíaca. Revista Mineira de Enfermagem, 16(3), 382-390.

Coppetti, L. D. C., Stumm, E. M. F., \& Benetti, E. R. R. (2015). Considerações de pacientes no perioperatório de cirurgia cardíaca referentes às orientações recebidas do enfermeiro. Revista Mineira de Enfermagem, 19(1), 113-126.

Costa, T. R. M., Florêncio, P. C. M., Castro, C. A. L., Ruiz, B. A., Souza, C. S., Barbosa, K. K. S., \& Barros, M. A. L. (2020). Complicações dos métodos de revascularização cardíaca em pacientes que sofreram infarto agudo do miocárdio. Revista Eletrônica Acervo Saúde, $12(11)$, e4834-e4834.

Galvão, M. C. B., Pluye, P., \& Ricarte, I. L. M. (2017). Métodos de pesquisa mistos e revisões de literatura mistas: conceitos, construção e critérios de avaliação. InCID: Revista de Ciência da Informação e Documentação, 8(2), 4-24.

Garcia, R. P., Budó, M. D. L. D., Schwartz, E., Simon, B. S., \& Silva, F. M. D. (2015). Apoio social frente à necessidade de cuidado após infarto do miocárdio. Revista Brasileira de Enfermagem, 68, 649-655.

Gomes, E. T., Silva, J. I. D., \& Bezerra, S. M. M. D. S. (2020). Elaboração da escala de avaliação do conhecimento de pacientes acerca da cirurgia cardíaca. Rev. SOBECC, 227-233.

Kahl, E. R. P. Y., Brião, R. D. C., Costa, L. M. D., Silveira, L. R., \& Moraes, M. A. P. D. (2019). Cenário ambulatorial de pacientes com sítio cirúrgico infectado após intervenção cardíaca. Revista Gaúcha de Enfermagem, 40.

Lima, V. R. D., Leal, C. C. G., \& Flávi, A. G. C. (2014). Complicações pós-operatórias em idosos submetidos à revascularização do miocárdio. CuidArte, Enferm, 48-54.

Lima, C. A., Richtrmoc, M. K., Leite, W. S., Silva, D. A. R. G., Lima, W. A., Campos, S. L., \& Andrade, A. D. D. (2019). Impacto do fast track em cirurgia cardíaca de adultos: desfechos clínicos e hospitalares. Revista Brasileira de Terapia Intensiva, 31, 361-367.

Lopes, R. O. P., de Castro, J., Nogueira, C. S. C., Braga, D. V., Gomes, J. R., da Silva, R. C., \& Brandão, M. A. G. (2019). Complicações do pós-operatório imediato de cirurgia cardíaca eletiva: estudo transverds. Revista de Enfermagem Referência, 4(22), 23-32. 
Research, Society and Development, v. 11, n. 2, e43611225920, 2022

(CC BY 4.0) | ISSN 2525-3409 | DOI: http://dx.doi.org/10.33448/rsd-v11i2.25920

Matioli, K. B. B., de Moraes Filho, I. M., de Sousa, T. V., Pereira, M. C., da Silva, R. M., de Sá, E. S., \& de Oliveira, M. L. C. (2021). Delirium: prevalência e fatores associados ao pós-operatório de cirurgia cardiovascular em idosos. Revista Baiana de Enfermagem, 35.

Menezes, T. C. D., Bassi, D., Cavalcanti, R. C., Barros, J. E. S. L., Granja, K. S. B., Calles, A. C. D. N., \& Exel, A. L. (2018). Comparações e correlações da intensidade da dor e da força muscular periférica e respiratória no pré e pós-operatório de cirurgia cardíaca. Revista Brasileira de Terapia Intensiva, $30,479-$ 486.

Paiva, A. C. R., Araújo, B. S., Carvalho, B. R., Arantes, D. C., Marinho, L. M., Silva, M. S., \& Moreira, L. R. (2015). Checklist de cirurgia segura: análise do preenchimento da ficha de verificação no pré, trans e pós-operatório. Enfermagem Revista, 18(2), 62-80.

Passinho, R. S., Sipolatti, W. G. R., Fioresi, M., \& Primo, C. C. (2018). Sinais, sintomas e complicações do infarto agudo do miocárdio. Rev. enferm. UFPE on line, 247-264

Paz, V. P., Fátima Mantovani, M., Mercês, N. N. A., da Silva, Â. T. M., \& Souza, P. B. (2019). Sistemas de cuidados à saúde de pessoas com infarto do miocárdio: revisão da literatura. Cogitare Enfermagem, 24.

Pereira, K. S. M., Oliveira, J. C. P. D., Carvalho, F. C. D., \& van Bellen, B. (2016). Complicações cardíacas em cirurgia vascular. Jornal Vascular Brasileiro, 15, 16-20.

Rabelo, A. C. S., Souza, F. V. F. S., \& Silva, L. D. F. D. (2018). Contribuição do cuidado transpessoal ao ser-cardiopata no pós-operatório de cirurgia cardíaca. Revista Gaúcha de Enfermagem, 38.

Reis, M. M. R., Lima, E. D. F. A., Casagrande, R. I., Fioresi, M., Leite, F. M. C., \& Primo, C. C. (2019). Perfil epidemiológico de pacientes submetidos a cirurgia cardíaca. Rev. enferm. UFPE on line, 1015-1022.

Ribeiro, C. P., Silveira, C.D.O., Benetti, E.R.R., Gomes, J.S., \& Stumm, E.M.F. (2015). Diagnósticos de enfermagem em pacientes no pós-operatório de cirurgia cardíaca. 9(2), 230-123.

Rosa, R. S., Macedo, D. A., Oliveira, B. G., Santos, B. E., Casotti, C. A., \& Prado, I. F. (2016). Evidências para o cuidado de Enfermagem na avaliação do Risco Coronariano em Pacientes Hospitalizados Evidence for nursing care in the evaluation of coronary risk in hospitalized patients. Revista de Pesquisa Cuidado é Fundamental Online, 8(2), 4460-4471.

Santos, J. D., Meira, K. C., Camacho, A. R., Salvador, P. T. C. D. O., Guimarães, R. M., Pierin, Â. M. G., \& Freire, F. H. M. D. A. (2018). Mortalidade por infarto agudo do miocárdio no Brasil e suas regiões geográficas: análise do efeito da idade-período-coorte. Ciência \& Saúde Coletiva, 23, 1621-1634.

Silva, C. J. B., Santos, S. M. É., Reis, F. F, Miranda, G. C. O., Santos, L., \& Lima, D. V. M. (2016). Banho no leito de infartados: crossover do controle hidrotérmico $40^{\circ} \mathrm{C}$ versus 42, 5. Revista Brasileira de Enfermagem Online, 15 (3), 341-350.

Silva, L. D. C., Melo, M. V. P., Rolim, I. L. T. P., \& Dias, R. S. (2018). Intervenções de enfermagem em pacientes de unidade de terapia intensiva cardiológica de um hospital universitário submetidos à cirurgia de revascularização do miocárdio. J Manag Prim Health Care [Internet], 9, e12.

Silva, L. L. T., Mata, L. R. F., Silva, A. F., Daniel, J. C, Andrade, A. F. L \& Santos, E. T. M. Cuidados de enfermagem nas complicações no pós-operatório de cirurgia de revascularização do miocárdio. Rev baiana enf. 31(3), 453-458.

Silva, M. T., Palu, L. A., \& Brusamarello, T. (2018). Prevenção de complicações evitáveis em uma unidade de terapia intensiva: uma revisão integrativa. Saúde e Pesquisa, 11(3), 613-621.

Silveira, E. L., Cunha, L. M., Sousa, P. M., Lima, A. V. M., \& Cunha, A. N. A. (2018). Prevalência e distribuição de fatores de risco cardiovascular em portadores de doença arterial coronariana no Norte do Brasil. Revista da Faculdade de Ciências Médicas de Sorocaba, 20(3), 167-173.

Smeltzer, S. C., \& Bare, B. G. (2014). Brunner \& Suddarth, Tratado de enfermagem médico-cirúrgica. In Brunner \& Suddarth, Tratado de enfermagem médico-cirúrgica (pp. 1133-1133).

Taurino, I. J. M. (2019). Cirurgia cardíaca: refletindo sobre o cuidado de enfermagem no período pós-operatório. Pub Saude, 13(5), 120-198. 